\title{
Estudio de las corrientes inducidas en bobina móvil pasando por un electroimán. Análisis de pérdidas por calor de joule
}

Felipe Garay ${ }^{1}$

\section{RESUMEN}

La idea de este trabajo nace de la publicación que John N. Fox y Daniel G. Reiber, hicieran en $1972,{ }^{2}$ cuyo objetivo principal era comparar la pérdida de energía cinética en un sistema de bobina móvil con la pérdida por calor por inducción de corrientes en esa bobina móvil: ambos efectos de pérdida se producen durante el tiempo que la bobina tarda en atravesar la zona magnetizada entre los polos de un potente electroimán.

Para comprobar esos efectos, consecuencia uno del otro, se coloca la bobina sobre un deslizador que descansa en un riel de aire. Este sistema deslizador-bobina se mueve sobre el riel de aire con fricción prácticamente despreciable: para que sea posible la minimización de fricción un compresor envía aire a muy alta presión y levanta ligeramente el sistema deslizador-bobina sobre el riel, de modo que este viaja realmente sobre un muy delgado colchón de aire, sin tocar el riel.

El mencionado artículo muestra que lo que no pudieron hacer entonces Reiber y Fox (por no contar en aquel entonces, a diferencia de nosotros, con un equipo ultrasensible de cuatro fotoceldas, un osciloscopio Tektronik de última generación y el software Mathematica 7.0), sí se pudo hacer ahora, al disponerse de esas herramientas, las cuales son clave para todo ello en el sustancial descenso del error. La reducción del error de un $13 \%$ (artículo mencionado) a $2 \%$ (el presente trabajo), permite estudiar posteriormente este mismo fenómeno, sustituyendo la bobina por una placa metálica para así analizar corrientes parásitas en la placa, corrientes similares a las inducidas en el caso de la bobina. El producto de esta investigación puede ser muy relevante a nivel industrial.

Palabras clave: levitación del anillo, inducción de corriente, electromagnetismo,

\footnotetext{
${ }^{1}$ Universidad Nacional Autónoma de Honduras. Facultad de Ciencias, Escuela de Física. Correo electrónico: felipe.jose.garay@gmail.com.

${ }^{2}$ Fox, John N and Reiber, Daniel G. Magnetic Induction and the Linear Track, Pennsylvania.
} 
disipación por el efecto de Joule.

\section{ABSTRACT}

The idea of this work derives from the publication that John N. Fox and Daniel G. Reiber, made in 1972 [1], whose main objective was to compare the loss of kinetic energy in a coil system with heat loss by the induced currents in the coil: both loss effects happened the time which the coil pass between the magnetized zone between the poles of a powerful electromagnet.

To check these effects consequence one from each other, the coil is placed on a slider cart resting on an air track. This slider cart-coil system moves air over the air track with negligible friction: for minimizing possible friction compressor sends air at very high pressure and slightly lifts the slider-coil system on the track, so that it travels on a very thin air bed, without touching the track.

The body of this article shows that what they could not Reiber and Fox do (for not having four photocells ultrasensitive equipment, a new generation oscilloscope, Tektronik, and Mathematica 7.0 software, in difference of our group) it could does now, providing us these tools, it was key of all in a substantial decrease of the error. The error reduction by $13 \%$ (before article) to $2 \%$ (our work) allowed us to study the same phenomenon subsequently replacing the coil with a metal plate to analyze eddy currents in the plate, similar to those induced currents in the coil. This future work may be very important for the Industry.

Key words: moving coil, induced current, electromagnet, dissipative joule losses.

\section{INTRODUCCIÓN}

El fenómeno que se estudia consiste en la inducción de corriente en una bobina. La Ley de Faraday justifica la inducción sobre cualquier circuito en el que el flujo magnético varíe con el tiempo. En nuestro caso, de las tres "opciones" de variación posible: campo magnético, área y ángulo, se trata de la segunda: el área que es atravesada por el campo magnético generado por el electroimán, varía a lo largo del recorrido que la bobina rectangular ejecuta en su entrada, permanencia y salida de la zona entre armaduras.

Esa pequeña corriente (registrada por un osciloscopio) absorbe la energía que 
necesita para ser producida, a expensas de la energía cinética de la bobina. Mientras que aquélla se disipa en calor por efecto Joule, la disminución de ésta provoca un frenado en el movimiento de la bobina.

El trabajo realizado permite ver la equivalencia entre la pérdida calorífica versus la de energía cinética.

\section{MARCO TEÓRICO}

Imagen 1. Sistema bobina móvil pasando a través del electroimán en un riel de aire

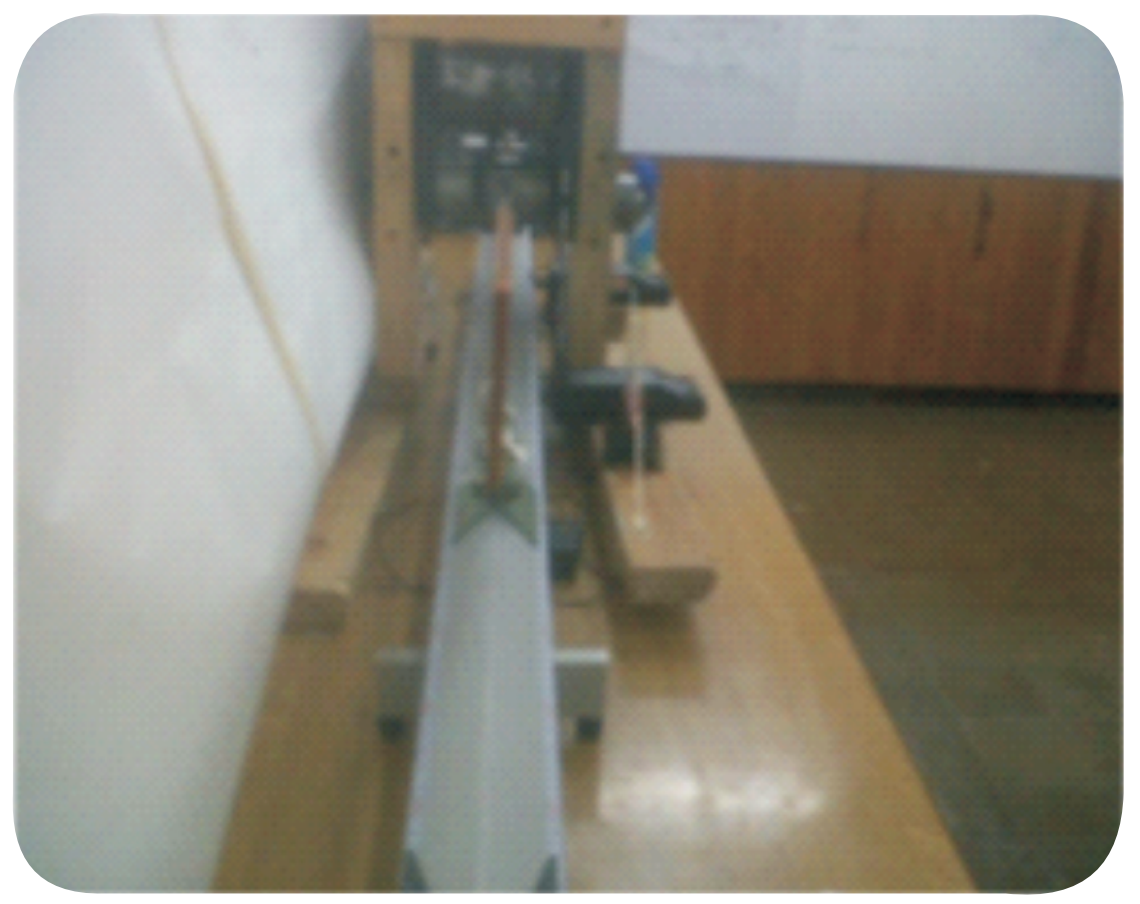

En una sección a lo largo del riel se instala el electroimán de tal forma que la bobina, colocada sobre el deslizador y arrollada en un soporte de madera, pueda atravesar la zona entre los polos del electroimán. El campo magnético que atraviesa la bobina produce un efecto de frenado ${ }^{3}$ debido a la pérdida de energía invertida en inducir las corrientes en la bobina; esto, como ya se sabe (algo básico en la teoría de la

${ }^{3}$ Ver: González y Hernández, Alejandro y Mora, César. (2010). Freno magnético. Departamento de Física, Facultad de Ciencias, UMAM. 
inducción electromagnética), ${ }^{4}$ ocurre primordialmente en el momento de entrada a la zona entre polos y en el momento de salida de esa zona.

Desde el punto de vista del fenómeno de inducción electromagnética, también es importante tener en cuenta la configuración no uniforme del campo magnético del electroimán (ver anexo) y que la bobina, que se mueve por traslación a través del electroimán, atraviesa toda la zona de campo no nulo.

La ecuación teórica que fundamenta el trabajo no es más que la ley de conservación de la energía, adaptada a nuestro sistema de bobina móvil, atravesando un campo magnético. Se trata de energía disipada en calor por la resistencia eléctrica de la bobina = pérdida de energía cinética de la propia bobina.

Esa pérdida de energía cinética que experimenta el sistema deslizador-bobina se debe, pues, esencialmente a la disipación resistiva de la bobina. ${ }^{5}$ Entonces, en este experimento se realizaron dos análisis:

1. Un análisis de la f.e. $m^{6}$ inducida $\left(V_{\text {ind }}\right)$ en la bobina (cuya energía asociada, como ya se ha dicho, se convierte en calor por disipación resistiva). ${ }^{7}$

2. Un análisis cinemático del sistema: que consiste en estudiar y medir la reducción de velocidad en las zonas en que la velocidad del sistema deslizador-bobina deja de ser constante, es decir, donde la acción del campo magnético es observable.

Con relación al primer análisis, el paquete de ecuaciones necesarias se describe a continuación. La potencia $\left(P=\frac{d W}{d t}\right)$ consumida por el sistema se debe a la disipación calorífica de la bobina, por efecto Joule, o sea:

$$
P_{\text {disipada }}=\frac{V_{\text {ind }}{ }^{2}}{R_{e Q}}
$$

como: $\quad P_{\text {disipada }}=\frac{d W_{\text {disipado }}}{d t}$ y $V_{\text {ind }}(t)$ es variable con el tiempo, (1)

se debe reescribir como: $\quad \frac{d W_{\text {disipado }}}{d t}=\frac{V_{\text {ind }}(t)^{2}}{R}$

\footnotetext{
${ }^{4}$ Ver Ref. 4c, pp. 332 a 340.

${ }^{5}$ Pérdidas de energía por calor debidas a la resistencia eléctrica de la bobina móvil.

${ }^{6}$ Fuerza electromotriz inducida (voltaje inducido en la bobina debido a la variación del flujo magnético).

${ }^{7}$ Se usó para ello un osciloscopio de última generación, Tektronix TDS2000, con precisión de micro voltios.
} 
De lo que finalmente se obtiene, al integrar: $W_{\text {disipado }}=\frac{1}{R} \int_{t_{0}}^{t} V_{\text {ind }}{ }^{2} d t$

Donde $R$ representa la resistencia de la bobina móvil.

Con respecto a la parte teórica relativa al segundo análisis, se puede observar que el teorema trabajo-energía cinética establece que el trabajo realizado sobre un sistema en movimiento acelerado es igual al cambio en la energía cinética de dicho sistema:

$$
W_{\text {disipado }}=-\Delta K
$$

Donde:

$$
\Delta K=\frac{1}{2} m\left(v_{2}^{2}-v_{1}^{2}\right)
$$

Como:

$$
\begin{gathered}
v_{1}=v_{\text {entrada }}=\frac{L}{\Delta t_{1}} y \\
v_{2}=v_{\text {salida }}=\frac{L}{\Delta t_{2}}
\end{gathered}
$$

La ecuación (4) se reescribe:

$$
\Delta K=\frac{1}{2} m L^{2}\left(\frac{1}{\left(\Delta t_{2}\right)^{2}}-\frac{1}{\left(\Delta t_{1}\right)^{2}}\right)
$$

Finalmente, combinando (2), (3) y (5):

$$
\frac{1}{R} \int_{t_{0}}^{t} V_{\text {ind }}{ }^{2} d t=-\frac{1}{2} m L^{2}\left(\frac{1}{\left(\Delta t_{2}\right)^{2}}-\frac{1}{\left(\Delta t_{1}\right)^{2}}\right)
$$

Esta última expresión (6) representa la conservación de energía aludida al principio y muestra el objetivo principal del trabajo: la comparación, ya aludida, del análisis cinemático con el de inducción magnética y el subsiguiente efecto, las pérdidas por calor producidas por efecto Joule sobre la pequeña resistencia de la bobina.

\section{DETALLES DEL TRABAJO EXPERIMENTAL}

Para el estudio cinemático se utilizaron un par de fotoceldas que detectan tiempo 
con precisión de décimas de milisegundo. Mediante el registro de ese tiempo (con la precisión dicha) se pudo conocer la velocidad de la bobina antes de entrar y después de salir de entre los polos del electroimán.

Se midió, como es lógico, la masa del sistema combinado (deslizador-bobina) con una balanza de buena y suficiente precisión. Se calculó así la pérdida de energía cinética entre los puntos donde el campo magnético es efectivo. Recuérdese que la pérdida por fricción es despreciable (el sistema se mueve con fricción casi nula por la acción del compresor en el riel que al levantar el deslizador lo mueve sobre un colchón de aire).

Imagen 2. Bobina rectangular de madera con 200 vueltas de cable magnético\#18

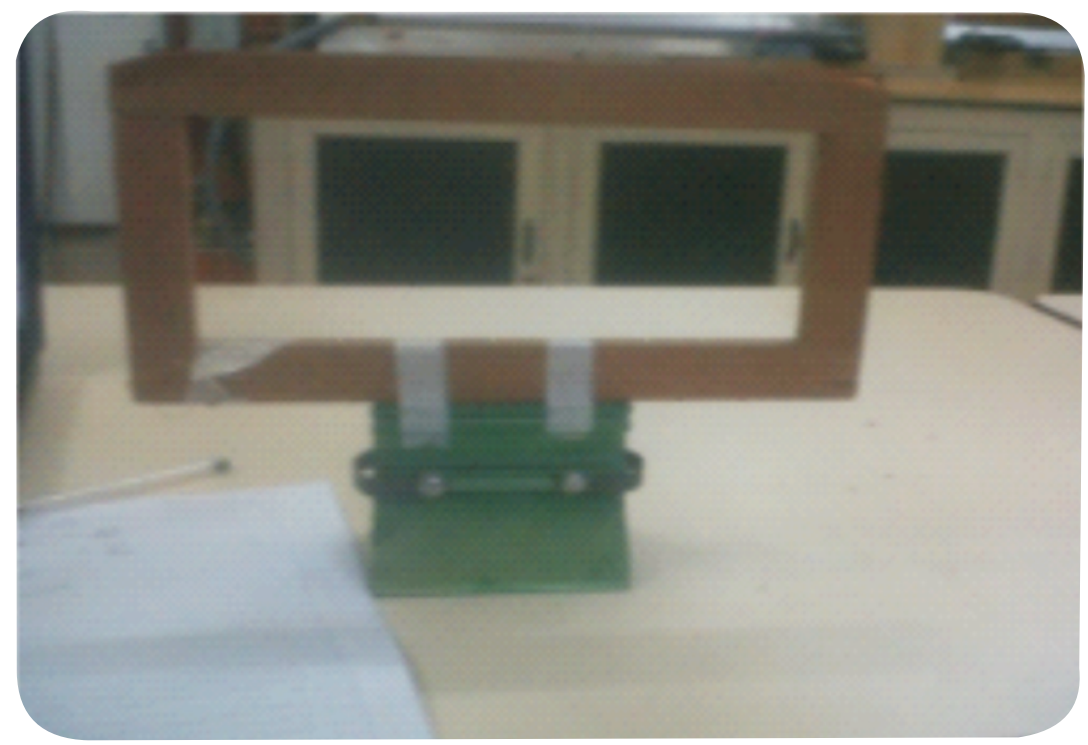

Las gráficas de variaciones de voltaje inducido en el tiempo, incluyendo toda la información correspondiente a esas variaciones, quedó almacenada en la memoria con que está dotado el osciloscopio. Luego se trasladaron a la computadora para ser procesadas y analizadas con el software Mathematica 7.0, con el fin de obtener un modelo matemático que se adaptara a los datos encontrados y con el cual se pudiera manejar el error y mejorar sensiblemente el nivel de exactitud.

Se midió también la longitud $L$ de la bobina y la diferencia en el tiempo, registrada por cada fotocelda cuando la bobina pasa a través de una y otra; esto permitió calcular las velocidades antes y después de que la bobina atravesara los 
polos del electroimán al cruzar toda aquella zona de campo efectivo, como ya se explicó anteriormente. Fuera de esa zona, el sistema deslizador-bobina se mueve con movimiento rectilíneo de velocidad constante.

\section{ANÁLISIS DE DATOS}

Para realizar los cálculos sobre las series de datos encontrados durante el proceso de medición en la bobina móvil, es necesario establecer un modelo matemático adecuado (ha de tratarse obligatoriamente de un modelo no lineal) para la función de voltaje inducido). ${ }^{8}$ En tal sentido, Mathematica 7.0 fue de gran utilidad (para el tiempo de Reiber y Fox apenas comenzaba el uso de computadoras, además, en aquella época eran bastante rudimentarias).

Con relación a la función de voltaje inducido, obtenida con el osciloscopio (ver gráfico 1), parecería que en principio se podría expresar mediante la suma de dos funciones de Gauss; una con coeficiente positivo y otra con coeficiente negativo. Ahora bien, las señales reales recibidas en el osciloscopio son demasiado estrechas: las curvas de voltaje inducido a la entrada y a la salida de entre los polos del electroimán se acercan más bien a pulsos. Entonces, la función gaussiana pura no cumple con las condiciones para modelar adecuadamente el fenómeno, por ello se utilizaron funciones tipo "delta de Dirac aproximada", estas son gaussianas 'estrechadas' por un factor multiplicativo; asimismo, esas funciones se definieron con cierto desfase temporal, pues los pulsos correspondientes no se encuentran centrados en $\mathrm{t}=0$.

En fin, la función de Dirac aproximada que empleada fue:

$$
\delta(t-c) \approx \pm \frac{a}{h^{2}} e^{\frac{-(t-c)^{2}}{2 h^{2}} 9}
$$

Registrada la señal de voltaje en el osciloscopio, mediante Mathematica $7.0,{ }^{10} \mathrm{se}$ hizo el tratamiento de datos que permitió modelar la función adecuada.

\footnotetext{
${ }^{8}$ De toda la data de la Tabla 1 (verla anexo), se tomó en el artículo la serie de la primera fila.

${ }^{9}$ Ver: Arfken, George B. y Weber, Hans J. Mathematical Methods for Physicists.

${ }^{10}$ Mathematica 7.0: software computacional avanzado de análisis de datos, reconocido y usado en las mejores universidades del mundo.
} 
Gráfico 1. Gráfica de los datos obtenidos de voltaje en función del tiempo ${ }^{11}$

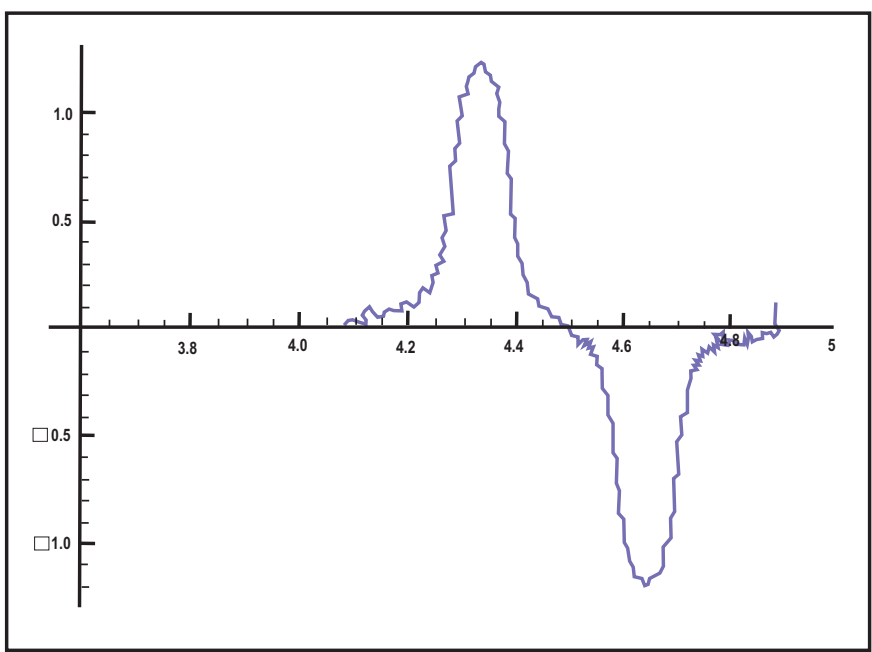

La función de voltaje construida mediante el referido ajuste (ver gráfico 2) es:

$$
V(t)=1.26\left[e^{-223.6(-4.3+t)^{2}}-e^{-229.3(-4.6+t)^{2}}\right]
$$

Gráfico 2. Función de voltaje inducido (V) vs. tiempo (ms), obtenida mediante ajuste no lineal.

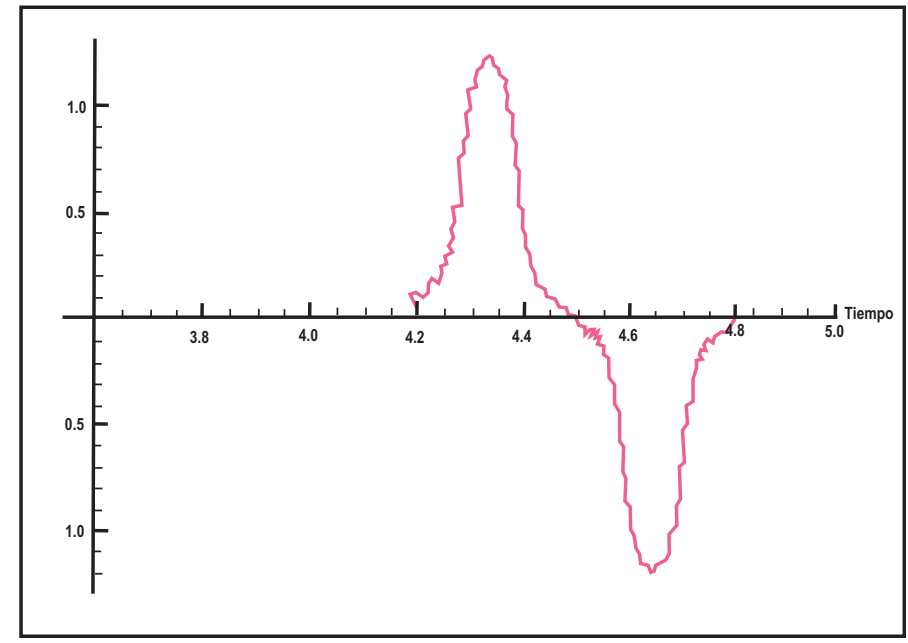

${ }^{11}$ Las funciones de voltaje inducido que aparecen en las figuras $3,4,5$ y 6 se refieren solo a la serie de datos de la primera fila de la tabla 1. 
Puede observarse a continuación (gráfico 3) que la función construida mediante el ajuste no lineal se acopla muy bien a los datos de función real de voltaje obtenida.

Gráfico 3. Superposición de los datos obtenidos (azul) y la función 'construida' mediante regresión no lineal (rojo).

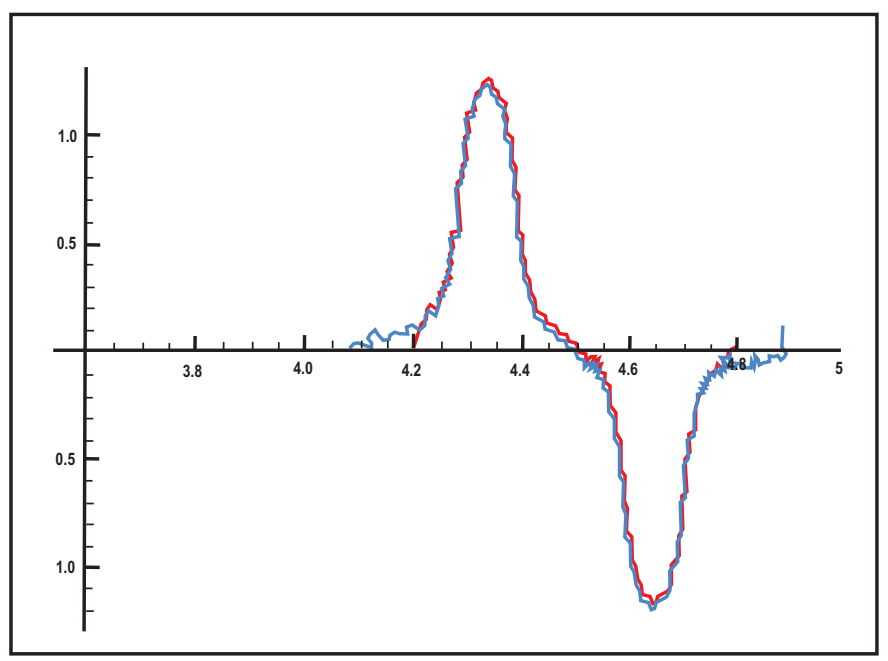

Para estimar las pérdidas por calor, se necesita $V_{\text {ing }}(t)^{2}$ (ver ec. (1): Ha de "elevarse al cuadrado" la función de (8), graficada en la gráfica 3. El resultado gráfico se ofrece en la gráfico 4.

Gráfico 4. Gráfica de la función voltaje cuadrado (Voltios ${ }^{2}$ ) vs. tiempo (ms)

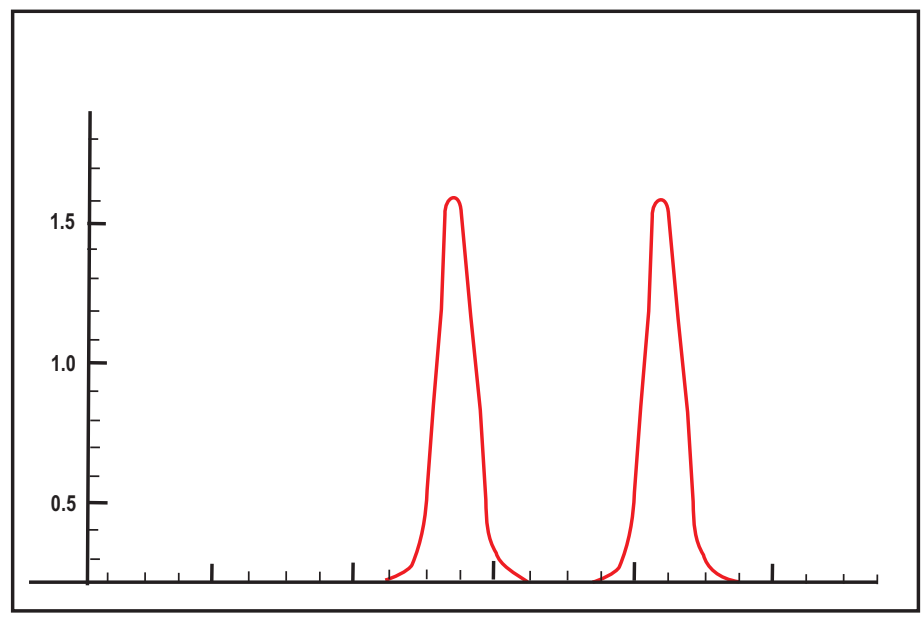


Sustituyendo en la ecuación (1), la función del modelo no lineal, dada en la ec. (8) y el valor de la resistencia, medido con un ohmímetro $\mathrm{R}_{\mathrm{eq}}=77.4 \Omega$ se obtiene (ver tabla 1, primera fila):

$$
Q=0.003365 \mathrm{~J}=3.365 \mathrm{~mJ}^{12}
$$

El cambio en la energía cinética, siempre para el caso de los datos de la primera fila de la Tabla 1 se obtiene sustituyéndolos en la ecuación (5), lo cual comporta:

$$
\Delta K=-0.003282 J=-3.282 \mathrm{~mJ}
$$

Tabla 1. Resultados obtenidos en distintos experimentos realizados para diferentes velocidades de entrada con la misma bobina móvil

\begin{tabular}{|c|c|c|c|c|c|c|}
\hline \multicolumn{5}{|c|}{ Análisis cinemático } & $\begin{array}{c}\text { Análisis } \\
\text { eléctrico }\end{array}$ & Comparación \\
\hline $\begin{array}{c}\text { Tiempo de } \\
\text { entrada (s) }\end{array}$ & $\begin{array}{c}\text { Tiempo de } \\
\text { salida (s) }\end{array}$ & $\begin{array}{c}\text { Velocidad } \\
\text { entrada (m/s) }\end{array}$ & $\begin{array}{c}\text { Velocidad } \\
\text { salida (m/s) }\end{array}$ & $\begin{array}{c}\text { Cambio en la } \\
\text { energía } \\
\text { cinética }(\mathrm{mJ})\end{array}$ & $\begin{array}{c}\text { Calor de } \\
\text { Joule }(\mathrm{mJ})\end{array}$ & $\begin{array}{c}\text { Porcentaje de } \\
\text { precisión de la } \\
\text { experiencia }\end{array}$ \\
\hline 0,4424 & 0,4507 & 0,6781 & 0,6656 & 3,2820 & 3,365 & 2,38 \\
\hline 0,3586 & 0,3619 & 0,8366 & 0,829 & 2,5160 & 2,481 & 1,41 \\
\hline 0,3146 & 0,3188 & 0,9536 & 0,941 & 4,6650 & 4,850 & 3,81 \\
\hline 0,2886 & 0,3107 & 1,0395 & 0,9656 & 28,957 & 30,637 & 5,48 \\
\hline 0,2063 & 0,2156 & 1,4542 & 1,3915 & 34,869 & 33,84 & 3,04 \\
\hline
\end{tabular}

\section{DISCUSIÓN DE RESULTADOS}

Como se puede fácilmente calcular, a partir de los resultados recién reseñados arriba e incluidos en la tabla 1, la diferencia $\Delta K$ vs $Q$ es de apenas $0.08 \mathrm{~mJ}$ que porcentualmente equivale $2.38 \%$.

Respecto a la primera experiencia de este tipo realizada por John N. Fox y Daniel G. Reiber, ${ }^{13}$ se mejoró sustancialmente el nivel de error (en el caso de ellos era cerca del $15 \%$ ); esto por el proceso de cálculo (ajuste exponencial -no lineal-, fácil de realizar ahora mediante Mathematica 7.0) y la toma de datos de los tiempos inicial y final (debido al uso de fotoceldas que miden en el orden de ms). Para asegurar aún

\footnotetext{
${ }^{12}$ Ver tabla 1, primera fila

${ }^{13}$ Fox, John N and Reiber, Daniel G. (1972). Magnetic Induction and the Linear Track. Pennsylvania.
} 
más la fiabilidad en la toma de datos y facilitar el análisis de los pulsos de voltaje de entrada y salida del entrehierro del electroimán, se mapeó el campo magnético producido por aquel (ver anexo).

Incidentalmente, dado que era necesario para el presente trabajo haber mapeado el campo magnético del electroimán utilizado, supone un resultado útil para trabajos conexos, pues muestra la configuración del campo magnético de electroimanes con polos redondos.

\section{CONCLUSIONES}

De un lado los resultados en sí mejoran experiencias anteriores de otros grupos debido a la altamente significativa reducción del error. De otro lado, el trabajo realizado con este tipo de equipo y la herramienta matemática del caso, es de gran importancia ulterior, pues ayuda a mejorar el estudio y manejo de las corrientes parásitas en placas metálicas.

Con la investigación realizada, apoyada en los resultados obtenidos, se espera modelar las trayectorias y el comportamiento de dichas corrientes parásitas de manera más precisa y válida que la habitual; las trayectorias que se presentan en la bibliografía y artículos especializados, consultados en este artículo, son cerradas -como es preceptivo en la teoría electromagnética-, pero la suposición de que sean más o menos elípticas no está demostrada. Experimentalmente podrían, en realidad, tratarse de curvas, aunque cerradas, mucho más complicadas.

Conocer las trayectorias reales de las corrientes parásitas es importante en general para mejorar aparatos, de uso bastante común, como los que se describen a continuación (todos ellos se fundamentan en la acción de las corrientes parásitas):

a. Sistemas de detección de metales (usados por agencias de seguridad), los que podrían mejorar su eficiencia en los sistemas de detección convencionales.

b. Hornos de inducción: el aumento en la capacidad de funcionamiento se debe esencialmente a la producción de calor debida a la disipación resistiva de corrientes parásitas.

c. Mecanismo estabilizador de balanzas mecánicas de precisión que utilizan corrientes parásitas para amortiguar las oscilaciones cuando se miden masas muy pequeñas.

d. Transformadores y motores eléctricos: en ellos es de suma relevancia la reducción del calor disipado debido a las corrientes parásitas generadas en los núcleos. 


\section{BIBLIOGRAFÍA}

Arfken, George By Weber, Hans J. Mathematical Methods for Physicists.

Fox, John N. and Reiber, Daniel G. (1972). Magnetic Induction and the Linear Track. Pennsylvania.

González y Hernández, Alejandro y Mora, César. (2010). Freno magnético. Departamento de Física, Facultad de Ciencias, UMAM.

Wangsness, Roald K. (2001). Campos electromagnéticos. México: Editorial Limusa

\section{ANEXOS}

Campo magnético creado por el electroimán, mapeado con un teslámetro de $0.1 \mathrm{mt}$ de precisión

Gráfico 5. Campo magnético del electroimán visto en dos dimensiones (eje vertical. Campo magnético en $\mathrm{mT}$, eje horizontal: distancia en $\mathrm{mm}$ ). El origen corresponde al punto central entre polos del electroimán.

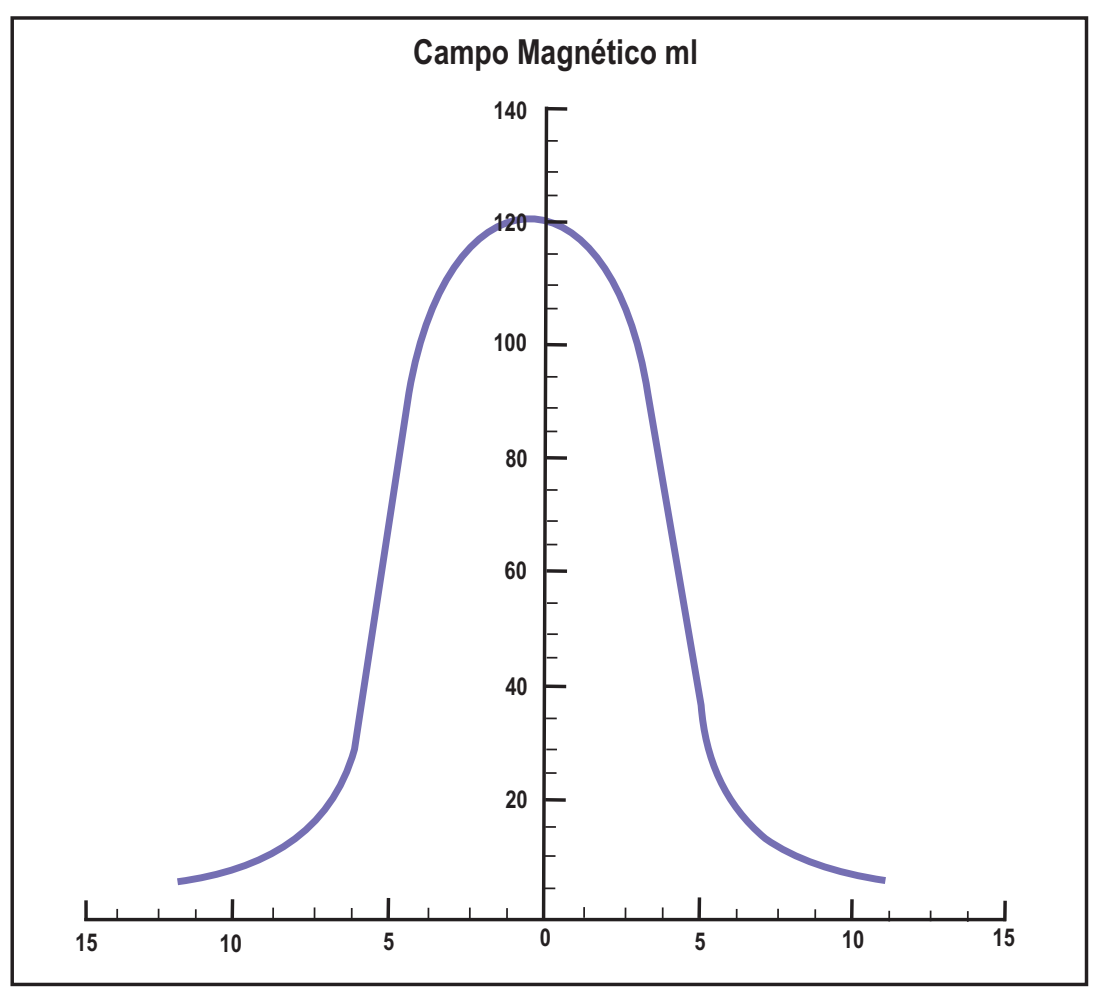


Gráfico 6. Campo magnético del electroimán visto en tres dimensiones

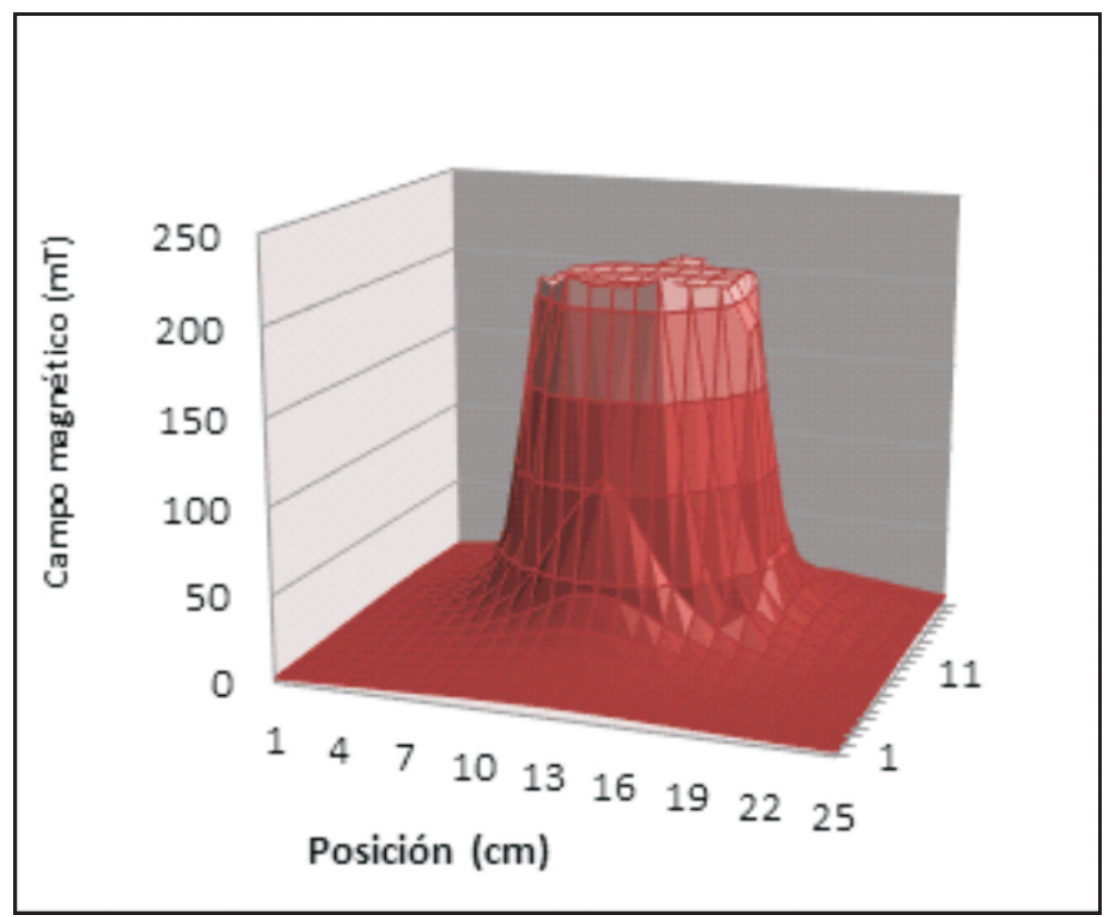

